\title{
OS DESAFIOS NA ATUAÇÃO DO TÉCNICO EM FARMÁCIA E DO FARMACÊUTICO EM FARMÁCIA CLÍNICA
}

\author{
THE CHALLENGES IN THE PERFORMANCE OF PHARMACY \\ TECHNICIANS AND PHARMACEUTICALS IN CLINICAL PHARMACY
}

\section{Henrique Sérgio Lima Gomes}

Acadêmico de Farmácia, Faculdade Presidente Antônio Carlos, Brasil

Email: henrique-sergio33@hotmail.com

Recebido: 10/06/2021 - Aceito: 19/06/2021

\section{RESUMO}

A assistência farmacêutica, dentro do cuidado básico do serviço de saúde unificado, é um índice de política pública de sua concepção em 1990. Mas apenas em 2004 se tornou regulatória. Esta assistência, que tem a medicina como uma contribuição essencial, visa o uso racional de drogas para garantir a melhoria da qualidade de vida. Tal regulamentação se mostra importante para o bom funcionamento das capacidades do profissional de farmácia no corpo dos atuantes na área da saúde. 0 farmacêutico é o profissional apto a realizar essa função, atuando no desenvolvimento, produção, manipulação, seleção e até mesmo aquisição, garantindo deste modo que as drogas sejam distribuídas de forma adequada. As intervenções realizadas pelo farmacêutico clínico são capazes de promover melhores resultados terapêuticos, garantindo a segurança e a eficiência. Este profissional em cuidados farmacêuticos fornece o uso racional de medicamentos, além de entrar em outros profissionais de saúde, para monitoramento adequado para o paciente em assistência médica. Sua função é essencial para o bom funcionamento não apenas das diversas áreas de saúde, mas mesmo de estruturas hospitalares, de modo a evitar o agravamento de problemas e ser um passo a mais rumo à busca pelo bemestar de pacientes. A evidência demonstrada neste estudo reforça a importância do serviço clínico de farmácia à conveniência e orientação do farmacêutico, bem como dos desafios enfrentados pelos técnicos de farmácia e farmacêuticos, apontando para as dificuldades que devem ser dirigidas no curso de sua profissão, abordando assim seu histórico, contexto global e nacional, assim como maiores agravantes, tais quais os efeitos da pandemia do COVID-19 na vida e na atuação de tais profissionais, de modo que se possa pensar em estratégias para que seja possível se rever não apenas a importância da profissão, mas meios de exercê-la da melhor forma, tanto para o público geral como para os próprios profissionais.

PALAVRAS-CHAVE: Desafios; Farmacêuticos; Técnicos; Farmácia; Clínico. 


\section{ABSTRACT}

Pharmaceutical assistance, within the basic care of the unified health service, is an index of public policy of its conception in 1990. But only in 2004 did it become regulatory. This assistance, which has medicine as an essential contribution, aims at the rational use of drugs to guarantee an improvement in the quality of life. Such regulation proves to be important for the proper functioning of the capacities of the pharmacy professional in the body of those working in the health area. The pharmacist is the professional able to perform this function, working in the development, production, handling, selection and even acquisition, thus ensuring that the drugs are properly distributed. The interventions carried out by the clinical pharmacist are capable to promoting better therapeutic results, guaranteeing safety and the efficiency. This professional in pharmaceutical care provides the rational use of medicines, in addition to joining other health professionals, for adequate monitoring for the patient in medical care. Its function is essential for the proper functioning of not only the different areas of health, but even of hospital structures, in order to avoid aggravating problems and being a further step towards the search for the well-being of patients. The evidence demonstrated in this study reinforces the importance of the clinical pharmacy service to the convenience and guidance of the pharmacist, as well as the challenges faced by pharmacy technicians and pharmacists, pointing to the difficulties that must be addressed in the course of their profession, thus addressing their history, global and national context, as well as major aggravating factors, such as the effects of the COVID-19 pandemic on the lives and performance of such professionals, so that strategies can be considered so that it is possible to review not only the importance of the profession, but means of exercising it in the best way, both for the general public and for the professionals themselves.

KEYWORDS: Challenges; Pharmacists;Technical; Pharmacy; Clinical.

\section{Introdução}

A farmácia hospitalar recebeu mudanças significativas nos últimos anos, mas coexistem várias realidades em nosso país, consistindo de farmácias extremamente modernas que oferecem toda a oferta de serviço aos outros extremos com hospitais sem fármacos. De acordo com Freitas et al (2016), o Brasil é "[...]um país continental e a farmácia hospitalar fica com vários desafios, por exemplo, com farmacêuticos em $100 \%$ dos hospitais. Além disso, deve-se melhorar a administração, ação que envolve armazenamento, planejamento, compras, etc.". Melhorar a qualidade técnica é outra urgência na entrega, manipulação, participação em grupos multiprofissionais e ações de farmácia clínica. Há progresso, especialmente com as diretrizes de ensino (MEC, 
2002); RDC 44/09 (ANVISA) com foco em serviços farmacêuticos; as decisões do Conselho Federal da Farmácia (CFF) - 585/13 e 586/13 - com a regulação das atividades clínicas e prescrições farmacêuticas.

Freitas et al (2016) também apontam para o fato de que a implementação do Sistema Único de Saúde (SUS) tornou possível definir a assistência farmacêutica e incluí-la como parte integrante da política de saúde pública. Essa inclusão foi possível, a totalidade, garantida pelo SUS, defende especial atenção aos seus usuários, estimulando a utilização de várias tecnologias capazes de promover a saúde da população, depois se tornar uma das bandeiras da saúde.

Apesar dos profissionais farmacêuticos devidamente treinados para 0 desenvolvimento, produção, manipulação, seleção e dispensação de drogas e terem várias áreas de atividade, como farmácias, indústrias, análises clínicas, monitoramento da saúde, unidades de saúde familiar (USF) de pesquisa ou Centros de educação, mesmo depois de mais de 20 anos de suspeita, a assistência farmacêutica no serviço de saúde pública em seus vários níveis de atenção é insuficiente, restritiva, muitas vezes, apenas em áreas de gestão de medicamentos e logística (FREITAS et al, 2016).

\subsection{Objetivos}

Este trabalho visa explorar os desafios que envolvem o ser farmacêutico ou técnico em farmácia no que diz respeito a sua atuação clínica, abordando as dificuldades que vêm com o exercício profissional. A importância deste estudo diz respeito a toda uma relação com as problemáticas que envolvem a multidão de atividades que são designadas a estes profissionais, buscando assim a possibilidade de um melhor curso de ação, além de medidas educativas que sirvam para instruir futuros farmacêuticos e técnicos em farmácia. 


\section{Revisão da Literatura}

\subsection{Farmácia Clínica no mundo}

A farmácia clínica vem dos hospitais e marcou oficialmente a final da fase tradicional e o início da fase de transição da profissão farmacêutica. O hospital foi comparado com as farmácias magistrais, para o lugar mais onde se realizariam atividades clínicas. No entanto, no hospital, este especialista também se afastou da manipulação de medicamentos e se aproximou da liderança, uma vez que a maioria dos medicamentos manipulados por farmacêuticos foram adquiridos como um produto acabado com a indústria farmacêutica. Com a introdução de farmacêuticos clínicos tinha ajuda com a equipe de pacientes e saúde como objetivo principal, que permitiu ao desenvolvimento de atividades clínicas, principalmente em termos de farmacoterapia (MENEZES, 2000).

De acordo com Amery (1999),o surgimento da farmácia clínica no ambiente hospitalar pode ser explicado às condições de trabalho do farmacêutico, uma vez que a característica da "entrega" ou o "distribuidor" do medicamento, possivelmente no hospital seja mais notável, onde geralmente a farmácia do hospital está em uma área que é removida das camas e, portanto, também dos pacientes e outros que são responsáveis pela atenção direta daqueles. Assim, o farmacêutico fornece a medicação somente nas quantidades e dosagens adequadas, de forma que a entrada do paciente em questão ocorra sem um monitoramento direto, o que também tem grande importância na distribuição de dietas industrializadas, pois está disponível a partir da farmácia, e há detalhes padronizados de acordo com a necessidade de cada paciente (ARRAIS et al, 2007). Esta estrutura de trabalho, na qual o profissional de farmácia encontrava-se responsável por um monitoramento de pacientes, permitiu que esses profissionais tivessem acesso a todas as receitas realizadas em hospitais, portanto, esta informação foi disponibilizada e desenvolveram-se novos serviços e estudos que demonstraram relevância em comparação com outros familiares de familiares no tratamento farmacológico de estadias (ALANO et al, 2012). 
Nos hospitais, devido à informação disponível nos regulamentos médicos e nas atividades disponíveis no ambiente hospitalar, seria entendido que as primeiras atividades clínicas dos farmacêuticos foram estudos relacionados à identificação de erros de prescrições, ocorrência de efeitos colaterais, efeitos de ingredientes ativos e iatrogenias (CUNHA, 1981. GOMES JÚNIOR, 1988).

De acordo com Cunha (1981), os resultados alcançados e a disseminação de tais trabalhos permitiram que se adquirisse o título de farmacêutico na Universidade de São Francisco, no estado da Califórnia, expandindo sua área de inserção ao ambiente hospitalar, onde viria a ter um reconhecimento gradual por outros profissionais de áreas correlatas. A partir de 1957, a American Hospital Association começou a incentivar outros farmacêuticos hospitalares nos Estados Unidos a desenvolverem estudos semelhantes aos desses grupos. Gradualmente, a farmácia clínica foi reconhecida em hospitais nos Estados Unidos (BELTON, 1997). Portanto, as faculdades farmacêuticas devem ser adaptadas a essa nova atividade, implementando em seus currículos de ensino, na disciplina de farmácia clínica, que deve ser integrada à formação teórica e ao desenvolvimento de argumentos clínicos com as academias de farmácia que estavam prontos para esta área. Para decidir o trabalho.

A este respeito, no final da década de 1960, os pesquisadores defenderam, como Mounier (1968), defendeu que a disciplina de Farmácia Clínica deveria ter principalmente atividades práticas, relacionadas especificamente à ação clínica de farmacêuticos. Além disso, Robert Miller (1968) explicou que eles podem formar o farmacêutico clínico para as universidades, eles devem considerar a farmácia clínica como em consideração:

[...] área do currículo farmacêutico que lida com a atenção ao paciente com ênfase na farmacoterapia, desenvolvendo uma atitude orientada ao paciente, tornando-se necessário desempenhar habilidades de comunicação interprofissional e com os pacientes, tendo como objetivos: aplicações clínicas dos conceitos farmacológicos; conhecimento sobre diagnósticos, principalmente quando relacionados à farmacoterapia; desenvolver habilidades de interação com o paciente e com outros profissionais; conscientizar 0 paciente de sua responsabilidade na utilização dos medicamentos; integrar os conhecimentos adquiridos; conscientizar os farmacêuticos de sua responsabilidade na farmacoterapia. (MILLER, 1968 pg. 180). 
A farmácia clínica foi reconhecida como uma atividade farmacêutica na Inglaterra em 1988. A Sociedade Europeia da Farmácia Clínica (ESCP) definiu a farmácia clínica como "especialidades de área de saúde que descrevem as atividades e serviços do desenvolvimento farmacêutico clínico e promocional do uso racional e apropriado de medicamentos e seus derivados "(Franklin et al., 2005).

\subsection{Farmácia Clínica no Brasil}

No Brasil, em 1996, foram criadas pela Sociedade Brasileira de Farmácia Hospitalar, as diretrizes que regiam padrões mínimos para a farmácia do hospital, incluiu aspectos direcionados à clínica. No entanto, a maioria dos hospitais brasileiros concentra os deveres de farmacêuticos em outras atividades (FREITAS et al, 2016).

Até 2000, as práticas relacionadas ao ensino da farmácia clínica no Brasil são consideradas raras e uma criação pioneira de um serviço, em Natal, em 1979, coordenada pelo professor Dr. Tarcísio José Palhano. O desempenho em farmácias de magistrados, que seriam uma oportunidade para o desenvolvimento do desempenho clínico do farmacêutico, foi bastante desacreditado pelos profissionais dos anos 1970 e 1980. Muitas tentativas de fortalecer essa prática e ensino ainda não produzem resultados satisfatórios, uma vez que nas universidades, historicamente, concentra-se na preparação de profissionais para agir nas indústrias farmacêuticas ou nos laboratórios clínicos (GONÇALVES, 1994).

Embora a terminologia da farmácia clínica seja de 1960, esse termo ainda pode ser considerado relativamente novo nos países em desenvolvimento. Khan (2011) relatou um exemplo dessa situação, alegando que no Paquistão havia mudanças na formação do farmacêutico do que em 2004, em algumas universidades através da modificação de estudos de graduação ao transferir a abordagem para aprender a orientação do paciente.

De acordo com o mesmo autor, métodos de ensino tradicionais, como metodologia bancária, segundo a qual o educador deposita seu conhecimento 
unilateralmente, não é suficiente para fornecer o conhecimento acadêmico necessário, o que permite que eles trabalhem facilmente no campo de farmácia Clínica (KHAN, 2011). Diante disso, alguns estudos mostraram que métodos de ensino não convencionais, como a análise de estudos e requisitos de caso, aumentam o interesse dos alunos da disciplina. Esses novos métodos permitem que os alunos adquiram novas habilidades, tornando-as mais participativas durante o processo de aprendizagem. No entanto, Gomes (1988) aponta que deve ser mencionado, que, aliados com este método de ensino, atividades práticas, supervisionadas por um preceptor, desde que seja uma farmácia clínica, pode melhorar a formação desses alunos, porque permite que os alunos dividam experiências diárias durante o cenário, preceptor mais experiente, além de usar esta nova metodologia de ensino para ajudar a orientação dos usuários do sistema de saúde.

Os atendimentos de Atenção Primária à Saúde (APS) foram projetados de modo que se tivesse capacidade de resolução de aproximadamente $80 \%$ das necessidades e problemas de saúde e serem frequentemente os principais meios de entrega livre de drogas à população. No entanto, as USFs não tem esse profissional como membro da equipe. Existem 15 farmacêuticos inseridos em APSs, através de núcleos de apoio à saúde familiar (NASF) e que só atuam em unidades integradas, ajudando mais de uma equipe de cada vez. Exemplificando, das 181 equipes de USF do município de João Pessoa/PB, apenas 29 são cobradas pelo profissional farmacêutico da NASF (a Secretaria de Saúde do Município de João Pessoa Verbales). Devido à falta de farmacêuticos ou técnicos de farmácia, profissionais de enfermagem - enfermeiros e técnicos em enfermagem, os quais historicamente se encontravam mais envolvidos na assistência farmacêutica do PA, sobrecarregaram suas missões (CAVALCANTI, et. Al, 2013).

Considerando que a distribuição de drogas pronuncia o profissional e que deve incluir a orientação do paciente sobre o uso apropriado do medicamento, determinando o Conselho Regional de Enfermagem, da Paraíba - COREN/PB, a dispensação dos medicamentos por técnicos e profissionais de enfermagem, no estado, foi proibida, sendo infratores aqueles que praticam tal função (COREN/PB, 2013). 


\subsection{Panorama atual da Farmácia Clínica}

Quando se trata de farmácia clínica, refere-se ao farmacêutico que trabalha diretamente com usuários de medicamentos. (CORREIA et al, 2017). Mas trabalhando com o paciente, é certo seguir certas regras, que foram definidas por mais de 300 anos de outras profissões, com ampla experiência da atenção direta do paciente. Trabalhar com o paciente envolve interferir com a vida dessas pessoas e, como resultado, assumir responsabilidades pelas profissionais. (FREITAS, 2016).

A primeira ação profissional é analisar os medicamentos do paciente e como ele dá ao processo de uso em sua vida diária (HEN, 2002). Esta avaliação também passa pelo conhecimento do indivíduo, seus hábitos e todos os seus problemas de saúde. O objetivo desta avaliação é verificar se o paciente tem um problema. Devese, portanto, ter uma clareza sobre os problemas que procuramos nos identificar apenas para resolvê-los. Depois de identificar o problema, o farmacêutico deve construir um plano de cuidados para resolver esse problema. É importante enfatizar que a solução do problema relacionada ao uso da droga pode envolver outros profissionais além do próprio farmacêutico e do próprio paciente. (HEN, 2002). Muitas vezes, o paciente tem vários problemas, que devem ser priorizados pelo farmacêutico que será resolvido durante o acompanhamento. Depois de ter implementado o plano de precaução, é responsabilidade do farmacêutico para recuperar esse paciente ou contatá-lo, avaliar os resultados de suas intervenções e farmacoterapias. Então, o ciclo está completo. Ou seja, o profissional identifica o problema, busca meios de resolvê-lo, e acompanha o indivíduo para avaliar os resultados de suas intervenções. (HEPLER, 2004)

Retomando um ponto anterior, deve-se almejar uma metodologia de trabalho para alcançar todo esse âmbito, a metodologia que rastreará esquemas de prática, ou comportamento como mencionado. Isso é essencial para ser aceito e respeitado como profissionais de assistência. 
Esses padrões de prática garantem que o profissional segue os parâmetros científicos e éticos para intervir na vida dos pacientes, em vez de criar seus próprios métodos sem descansar a literatura. Em outras palavras, é necessário avaliar o paciente com base no conhecimento científico, no que já foi comprovado, de preferência em milhares de pessoas. Não se deve "inventar a roda". Somente assim o farmacêutico será respeitado pelos outros membros da equipe de saúde e será pago pelas decisões que ele leva (CORREIA et al, 2017).

São feitos esforços para a mudança do modelo de diretiva pedagógica atual, como avaliações de educadores em farmácia clínica em todo o país, realizadas em 2015 e 2016, que geraram uma matriz de concorrência para o produto farmacêutico clínico, o que permite o aumento da inserção do cuidado médico do eixo na nova proposta de um guia nacional de curso de farmácia enviada ao Conselho Nacional de Educação e a criação da Sociedade Brasileira de Farmácia Clínica (CORREIA et al, 2017).

Importa referir que a atividade clínica não pode ser realizada apenas do ponto de vista técnico e científico, sendo esta também a dificuldade elencada, devendo ser realizada compreendendo o indivíduo como um todo numa perspectiva humanizada e holística. O local onde ele viver afetará sua saúde. Outro ponto levantado por este estudo é que é difícil para o farmacêutico se reconhecer como profissional de saúde e / ou clínico (ALANO et al, 2012).

O fato de a indústria farmacêutica possuir um amplo leque de possibilidades de atuação também é considerado uma dificuldade para a realização da atividade clínica. De acordo com a Comissão Federal de Farmácia, os farmacêuticos têm 72 possibilidades. São tantas as atribuições, as possibilidades são diferentes e o foco clínico e a atuação de ser profissional de saúde estão perdidos. Gestores e falta de tempo também são considerados obstáculos importantes. (FREITAS et al, 2016). Em comparação com outras atividades a serem realizadas, a legislação sobre medicamentos envolve mais requisitos para os profissionais da empresa e a responsabilidade pelo gerenciamento especial de produtos de controle. Além dos aspectos quantitativos do controle de estoque, os gerentes costumam acreditar que os profissionais são necessários apenas para a tomada dessas decisões. 
As necessidades do paciente envolvem prestar atenção à sua saúde, e podem esclarecer dúvidas sobre os medicamentos empregados na terapia, tendo seus problemas desafiados. O profissional pode também dar orientações nãofarmacológicas, direcionadas aos benefícios de saúde do paciente que ainda são devido a outros remanescentes de profissionais, quando necessário. (HONG et al, 2009). Favorecer a adesão de pacientes com pacientes com pacientes é uma das principais funções que enfrentam serviços de saúde.

Foi realizado um estudo com 27 pessoas hipertensas no estabelecimento da farmácia popular em Alfenas/MG, que identificou que as razões para a não adesão ao tratamento foram: Esquecendo as diretrizes médicas (67\%), o uso da medicina em anão intencionalmente (53\%) e a falta de compreensão do uso de terapia medicamentosa (13\%) (Amarante, et al., 2010).

Em outra pesquisa clínica com 96 pacientes com diabetes mellitus tipo 2, realizou sistemas de saúde da comunidade privada dos sistemas de saúde do Paraná, constatou-se que era mais parte do problema relacionado ao medicamento encontrado em eficácia não terapêutica (68.1\%), à insegurança de medicamentos prescritos (16. $8 \%$ ), e para o problema, a necessidade de tratamento ou uso de medicamentos (15. 1\%) (CORRER, et., Al., 2009). É estimado que um grande número de dependentes não tem acesso ao número de medicamentos e à qualidade necessária para a eficácia terapêutica devido à dobragem na gestão de assistente técnico da dosagem. Por outro lado, a influência da indicação automática da mídia, para o uso abusivo dos produtos, intoxicantes, sem indicação clínica e/ou neutra, aumenta o acesso abusivo dos medicamentos, o que causa o crescimento dos convenientes problemas para eles. $O$ impacto desses programas é evidente em sistemas de saúde altamente subnotificados. Algumas instituições de saúde buscam promover o uso racional de medicamentos e resultados farmacoterapêuticos para realizações de ações de atenção de saúde referentes à população (CORRER, et., AL., 2011).

\subsection{Atuação do Farmacêutico}


O uso racional de medicamentos ocorre quando o paciente utiliza medicamentos para suas necessidades clínicas, obedecendo adequadamente a dose e a duração do tratamento, além de obter o produto farmacêutico por um custo acessível. A população educacional sobre o uso de medicamentos é uma parte fundamental para uso racional, e faz parte do cuidado farmacêutico. Para garantia do uso medicamentoso adequado, é necessário melhorar as ações e expandir a área de abrangência farmacêutica (MOTA, et al., 2008).

A incorporação de gestão clínica e técnica para atenção farmacêutica é importante para obter serviços mais eficientes nos processos derrotados à saúde, onde o aspecto clínico do desconforto pode contribuir para a otimização da ação da medicina e da terapia, com o objetivo de as reais necessidades dos pacientes serem atendidas, permitindo melhores resultados de saúde (CORRER, et., al., 2011).

O uso de medicamentos, sem a devida orientação, pode causar efeitos prejudiciais temporários ou até irreparáveis quando aplicado o tratamento terapêutico fornecido. A existência do profissional de farmácia (Farmacêutico ou técnico em farmácia) pode antecipar a manutenção e a recuperação da negação da saúde inserida em terapias medicamentosas (AMERY, 1999; ARRAIS et al, 2007; ALANO et al, 2012).

Devido à crescente complexidade das drogas, serviços farmacológicos têm sido cada vez mais necessários com indicações específicas relativas à utilização de remédios correspondentes, bem como com outras intervenções farmacêuticas, que beneficiam pacientes para que se possa obter uma melhor qualidade de vida (VINHOLES, et., al., 2009). Os serviços de farmácia clínica são divulgados diretamente do farmacêutico, e este serviço pode ser realizado em: hospitais, drogarias com salas devidamente separadas para tal, unidades sanitárias, domicílio ou educação de pacientes em que o indivíduo está exposto ao uso de drogas (GOMES, et al., 1988). O profissional de farmácia integrado na saúde multidisciplinar é responsável por quaisquer intervenções oriundas do uso medicamentoso seguro. Devido à casa que as necessidades dos pacientes não limitam o acesso ao fármaco, 
mas precisam de tratamento farmacêutico para a terapia medicamentosa, para que possam parecer ações para um evento adverso e uma solução inter-compatível de outros problemas causados por medicamentos (REIS, et . Al., 2013).

Tratamentos farmacêuticos exigem uma prática clínica profissional e um processo de assistência farmacêutica definido para que ele atenda aos seguintes propósitos (CORRER, et. , Al. , 2011): Fornecer termos administrativos clínicos e critérios para aplicar os farmacêuticos diretamente ao paciente em um físico estabelecido espaço; Assistência farmacêutica com pacientes, individualizados ou coletivamente, sobre necessidades precisas dos usuários de drogas, com o apoio da gestão técnica de serviços e gestão clínica do medicamento; serviços organizados para a situação epidemiológica do fármaco da Comunidade e para problemas de saúde individuais dos pacientes, isto é, é necessário conhecer a população em termos de fatores epidemiológicos, socioeconômicos, demográficos e ecológicos, bem como a entrega de medicamentos; planejar ações de educação em saúde para pacientes orientados para os farmacêuticos, particularmente em usuários medicinais em quantidade (polifarmácia) e uso contínuo, doenças crônicas, e isso é necessário para melhor experimentar sua doença e conformidade com as vezes drogas e lugares adequados para o seu tratamento (FREITAS, 2016).

Por outro lado, as ações clínicas são direcionadas para atendimento médico e resultados terapêuticos obtidos por meio de atividades no processo de saúde do paciente, levando em conta o farmacêutico como um profissional fundamental para obter bons resultados farmacoterapêuticos (SOLER, et. Al., 2010). Foi demonstrado que o desempenho do farmacêutico clínico colabora positivamente para a melhoria da saúde do paciente, uma vez que há um monitoramento contínuo de problemas de saúde, ambas as doenças crônicas e em problemas relacionados a medicamentos, isto é, o farmacêutico em um acompanhamento clínico aprende, identifica e resolve problemas de saúde, através de intervenções farmacêuticas (MIRANDA, et., 2012).

Um estudo retrospectivo, realizado na primeira unidade de serviço (UPA) no Morumbi do Hospital Israelense Albert Einstein (HIAE), demonstrou o desempenho e a importância do farmacêutico clínico, através da identificação, classificação e levantamento do número de intervenções realizadas (CORREIA, 2017). 


\subsection{Farmácia Clínica durante a pandemia de Covid-19}

Recentemente, a situação clínica referente à pandemia do Covid-19 criou muitas dificuldades e forçou a comunidade farmacêutica a examinar/personalizar processos essenciais para apoiar em um hospital (MEIRELES, 2020). Acredita-se que existem muitos desafios que alguns enfrentam como a técnica de correntes de serviço e rotinas, incluindo a entrega de medicamentos e elementos hospitalares, destacados para garantir que os produtos que resignam pacientes sem risco de contaminação e os usassem, poderia retornar aos inventários que obedecem aos critérios de segurança, como desinfecção, por exemplo, por proteção de relatórios saudáveis, desenvolvendo suas ordens (ANAMT, 2020).

Muitos hospitais escolheram a adaptação da infraestrutura física, criando/adaptando farmácias via satélite em ambientes isolados para cuidados específicos de áreas que receberam casos tranquilos suspeitos ou confirmados. O trabalho de biossegurança é cuidadosamente e intensamente realizado, com farmácias e outros funcionários, que constituem a farmácia hospitalar, especialmente se houver diretamente na participação direta com tais rios, e a utilização de equipamentos de proteção individual (EPI) e o uso de higiene manual de soluções antissépticas (ANAMT, 2020). O serviço clínico do profissional de farmácia em valorização e monitoramento de fármacos visando o tratamento de uma doença completamente nova é, sem dúvida, um dos maiores desafios em um cenário, nos estudos de eficiência clínica e se os riscos ainda são indecisos ou não. Neste contexto, o monitoramento de medicamentos é extremamente necessário, porque é possível cumprir reações adversas, alterações de medição, alterações dominantes laboratoriais que exigem uma aparência cuidadosa, em particular, focada na segurança do paciente (MEIRELES, 2020).

Outro problema que representa um dos desafios mais importantes está relacionado à provisão de medicamentos essenciais para cuidar de cuidados intensivos, principalmente a sedoanalgesia e o bloqueio neuromuscular, que afetaram 
Revista Multidisciplinar do Nordeste Mineiro, v.2,

$2021 / 02$

ISSN 2178-6925

todo o país, considerando uma enorme demanda. Este cenário exigiu aos serviços de farmácia esforços adicionais, de modo a suprir uma necessidade de alternativas terapêuticas durante $\mathrm{o}$ trabalho entre as equipes multidisciplinares, atuando nos serviços de manutenção e gerenciamento de suprimentos. (PEREIRA E FREITAS , 2008.) 


\section{Considerações Finais}

Este estudo levanta uma série de dificuldades relacionadas à mudança na concentração da profissão farmacêutica no país. A educação farmacêutica aparece como a barreira principal, seguida por uma desvalorização de uma profissão que envolve tamanhas responsabilidades. No contexto atual em que a educação está no país, cuida de uma abordagem quantitativa e produtos básicos, torna-se mais e mais profissional desqualificado, que não são reconhecidos como profissionais de saúde e com um baixo nível de conhecimento, afetando desempenho de serviços farmacêuticos. Problemas estruturais também foram identificados e, de fato, constituem um obstáculo à realização do trabalho clínico pelo farmacêutico. Dado que os gerentes, a equipe de saúde e a empresa ainda não estão convencidos de que esse profissional pode ser útil para resolver os problemas de saúde exigidos pelo sistema.

A profunda reforma educacional na formação técnica, na graduação e na pósgraduação é necessária para a mudança de paradigma, bem como de mudanças e como ensinar e como treinar habilidades. Os profissionais envolvidos na formação de farmacêuticos devem ser levantados sobre a necessidade de educação clínica de qualidade através de metodologias validadas a isso. Mais linhas de pesquisa na área devem ser encorajadas a estimular a formação de mais educadores em uma farmácia clínica. Autoaprendizagem como uma atividade da saúde profissional é essencial para o profissional de farmácia incorporar a filosofia da saúde e usar seu conhecimento para realizar atividades clínicas e se tornar diferencial dentro da equipe de saúde. No entanto, o profissional, que já está fazendo, precisa procurar capacidades e capacidades para o trabalho clínico e, em seguida, inseri-lo em prática, para mostrar seu potencial para administradores, outros profissionais de saúde e a comunidade.

Sem dúvida, relacionamentos e processos indispensáveis para a gestão dessa área devem ser integrados nos outros serviços de apoio, bem como nos participantes. "Pensamento", na ajuda farmacêutica, requer padrões de processo, fluxos, protocolos para uso efetivo, medicamentos seguros e produtos de saúde que sempre levam em 
conta a interface com outros hospitais e seguram o acesso a essas tecnologias de saúde.

\section{REFERÊNCIAS}

ALANO, G.M.; CORRÊA, T.S.; GALATO, D. Indicadores do Serviço de Atenção Farmacêutica (SAF) da Universidade do Sul de Santa Catarina. Ciência\&SaúdeColetiva, v.17, n. 3, 2012. p. 757-764.

AMARANTE, L. C. et al. A influência do acompanhamento farmacoterapêutico na adesão à terapia anti-hipertensiva e no grau de satisfação do paciente. Revista de Ciências Farmacêuticas Básica e Aplicada, [S.I.], v. 31, n. 3, p. 209-215, 2010.

AMERY, W. K. Why there is a need for pharmacovigilance? Pharmacoepidemiology and Drug Safety, v. 8.1999. p. 61-64,.

ANAMT. Associação Nacional de Medicina do Trabalho. Pandemia destaca os desafios enfrentados por profissionais de saúde em todo o mundo. Disponível em https://www.anamt.org.br/portal/2020/09/23/pandemia-destaca-os-desafiosenfrentados-por-profissionais-de-saude-em-todo-o-mundo/ (acesso 21/02/2021)

ARRAIS, P. S. D.; BARRETO, M. L.; COELHO, H. L. L. Aspectos dos processos deprescrição e dispensação de medicamentos na percepção do usuário: estudo de basepopulacional em Fortaleza, Ceará, Brasil. Cadernos de Saúcostde Pública, Rio de Janeiro, v. 23, n. 4, 2007. p. 927-937.

BELTON, K. The European Pharmacovigilance Research Group.Attitude survey ofadverse drug- reaction reporting by health care professionals across the European Union.Eur. J. Clin. Pharmacol, v. 52, 1997. p. 432-427.

CAVALCANTI, Ednamarah Luana de Medeiros; FREITAS, Raphael Alves de; BORBA, Ana Karoline Targino; FARIAS, Regina Lúcia Guedes Pereira de; AGUIAR, Fernanda Burle de; HIRSCH-MONTEIRO, Cristine. Desafios para a assistência farmacêutica na atenção básica à saúde. Centro de Ciências da Saúde/ Departamento de fisiologia e Patologia/ PROBEX. 2013

COREN/PB. PARECER COREN-PB № 092/2013. 2013.

CORREIA, Kleinia Karine de Lima; BARROS, Maria Luiza Carneiro Moura Gonçalves Rego; BARROS JÚNIOR, Marconi Rego; MARQUES, Ronmilson Alves. Farmácia Clínica: Importância deste serviço para o cuidado com a Saúde. Boletim Informativo Geum, v8, n3, 2017, p-7-18.

CORRER, C.J.; OTUKI, M.F.; SOLER, O. Assistência farmacêutica integrada ao processo decuidado em saúde: gestão clínica do medicamento.RevPanAmazSaude. v. 2, n. 3, , 2011. p.41-49.

CORRER, C.J.; PONTAROLO, R.; WIENS, A.; ROSSIGNALL, P.; MELCHIORS, A.C.; RADOMINSKI, R. Avaliação econômica do seguimento farmacoterapêutico em pacientes com diabetes melito tipo 2 em farmácias comunitárias. ArqBrasEndocrinalMetab. .2009. P.53-75 
CUNHA, B. C. A. Medicamentos: fator de saúde? São Paulo: Artpress, 1981.

FRANKLIN, $\quad$ B. $\quad$ D.; VAN MIL, J. Definingclinicalpharmacyandpharmaceuticalcare. Pharm World Sci, v. 27, n. 3, p. 137, 2005.

FREITAS, Gabriel Rodrigues Martins de; PINTO, Rodrigo Silveira; LUNA-LEITE, Márcia Dos Angeles; CASTRO, Mauro Silveira de; HEINECK, Isabela. Principais dificuldades enfrentadas por farmacêuticos para exercerem suas contribuições clínicas no Brasil.PPGCF/ Universidade Federal do Rio Grande do SUL (UFRGS). 2016.

GOMES-JÚNIOR, M. S. ABC da Farmácia. São Paulo: Org. Andrei, 1988. 215 p.

GONÇALVES, R. B. M. Tecnologia e organização social das práticas de saúde.São Paulo:HucitecAbrasco, 1994. 126 p.

HEN et al. Case studies in practice. Medication review: a process guide for pharmacists.Pharmaceutical Society of Australia, 2002.

HEPLER, C. D. Clinical Pharmacy, Pharmaceutical Care, and the Quality of Drug Therapy.Pharmacotherapy, v. 24, n. 11, 2004. p. 1491-1498.

HONG, A.L.; BROZICK, A.; LAM, S.; PARRIS, M.; PAINE, M.; FLOWERS, P.W. Pharmacist implementation in the emergency department.Ann Emerg Med. v. 54, n. 3, 2009.p.S78-9.

Khan N, Nakajima N, Wanderburg WH, compiladores. Healthy Work: an annotated bibliography. Lanham (MD): Scarecrow Press, Inc.; 2011. 376 p. 617.

MEIRELES, Gabriela. Profissionais da linha de frente encaram desafios de saúde mental na pandemia.2020. Disponível em https://www.medicina.ufmg.br/profissionais-da-linha-de-frente-encaram-desafios-desaude-mental-na-pandemia/ (acesso 21/02/2021).

MENEZES, E.B.B. Atenção farmacêutica em xeque.Rev. Pharm. Bras., v.22, n.2000. p.28.

MILLER, Robert. A farmácia clínica. Tradução de Fernanda Queiroz. São Paulo: IBRASA, 1968.

MIRANDA, J.O. Thermal conductivity of nanocomposites with high volume fractions of particles, Composites Science and Technology. México 2012

MOTA D.M., SILVA M.G.C., SUDO E.C., ORTÚNV. Uso racional de medicamentos: uma abordagem econômica para tomada de decisões. Ciênc Saúde Coletiva. v.13, p.S589-601, 2008.

MOUNIER, Emmanuel. O Personalismo. São Paulo: Centauro, 1968. 
PEREIRA, Leonardo Régis Leira; FREITAS, Osvaldo de. A evolução da Atenção Farmacêutica e a perspectiva para o Brasil. Rev. Bras. Cienc. Farm. vol.44 no.4 São Paulo Oct./Dec. 2008.

REIS W.C.T., SCOPEL C.T., CORRER C.J., ANDRZEJEVSKI V.M.S. Análise das intervençõesde farmacêuticos clínicos em um hospital de ensino terciário do Brasil. Einstein. v. 11, 2013. p.190-196.

SOLER, O.; ROSA, M.B.; FONSECA, A.L.; FASSY, M.F.; MACHADO, M.C.; SILVA, R.M.C. Assistência farmacêutica clínica na atenção primária à saúde por meio do Programa Saúde da Família. Rev. Bras. Farm. v.9, 2010. p. 37-45

VINHOLES, E.R.; ALANO, G.M.; GALATO, D. A percepção da comunidade sobre a atuação do serviço de atenção farmacêutica em ações de educação em saúde relacionadas à promoção do uso racional de medicamentos. Saúde Soc. v. 18, n.2. , 2009. p. 293-303. 